\title{
Streamlined genetic education is effective in preparing women newly diagnosed with breast cancer for decision making about treatment-focused genetic testing: a randomized controlled noninferiority trial
}

\author{
Veronica F. Quinn, BAHons, PhD'1, Bettina Meiser, BAHons, PhD'1, Judy Kirk, MBBS, FRACP2,3, \\ Kathy M. Tucker, MBBS, FRACP4, Kaaren J. Watts, BAHons, PhD ${ }^{1}$, Belinda Rahman, MGenCouns ${ }^{1}$, \\ Michelle Peate, MScMed(RH\&HG), PhD ${ }^{1,5}$, Christobel Saunders, MBBCh, FRACP 6 , \\ Elizabeth Geelhoed, PhD7, Margaret Gleeson, GradDip(GenCouns) ${ }^{8}$, Kristine Barlow- \\ Stewart, MGenCouns, PhD ${ }^{9}$, Michael Field, MBBS, FRACP ${ }^{10}$, Marion Harris, MBBS, FRACP ${ }^{11}$, \\ Yoland C. Antill, BMed, MD ${ }^{11,12}$, Linda Cicciarelli, MGenCouns ${ }^{13}$, Karen Crowe, MGenCouns ${ }^{14}$, \\ Michael T. Bowen, BScHons, PhD ${ }^{15}$, Gillian Mitchell, FRACP, PhD ${ }^{13,16}$; for the TFGT Collaborative Group
}

Purpose: Increasingly, women newly diagnosed with breast cancer are being offered treatment-focused genetic testing (TFGT). As the demand for TFGT increases, streamlined methods of genetic education are needed.

Methods: In this noninferiority trial, women aged $<50$ years with either a strong family history $(\mathrm{FH}+)$ or other features suggestive of a germ-line mutation (FH-) were randomized before definitive breast cancer surgery to receive TFGT education either as brief written materials (intervention group (IG)) or during a genetic counseling session at a familial cancer clinic (usual-care group (UCG)). Women completed self-report questionnaires at four time points over 12 months.

Results: A total of 135 women were included in the analysis, all of whom opted for TFGT. Decisional conflict about TFGT choice (primary outcome) was not inferior in the IG compared with the UCG (noninferiority margin of -10 ; mean difference $=2.45$; $95 \%$ confidence interval $-2.87-7.76 ; P=0.36)$. Costs per woman counseled in the IG were significantly lower (AUD\$89) compared with the UCG $($ AUD $\$ 173 ; t(115)=6.02 ; P<0.001)$.

Conclusion: A streamlined model of educating women newly diagnosed with breast cancer about TFGT seems to be a cost-effective way of delivering education while ensuring that women feel informed and supported in their decision making, thus freeing resources for other women to access TFGT.

Genet Med advance online publication 29 September 2016

Key Words: $B R C A 1$; $B R C A 2$; genetic counseling; psychological adjustment; rapid testing

\section{INTRODUCTION}

Women with breast cancer and a family history that includes multiple cases of breast and/or ovarian cancer may be referred to familial cancer clinics (FCCs) for genetic assessment and consideration of testing for germ-line mutations in cancer predisposition genes, including BRCA1 and BRCA2 (BRCA1/2). Genetic information provides the context for advice regarding women's personal future cancer risk and its management, helps to inform treatment decisions regarding their current cancer diagnosis, and provides risk estimations for unaffected blood relatives. ${ }^{1}$

Historically there has been little urgency for a genetic assessment at initial cancer diagnosis because the outcomes have generally not influenced cancer management in an individual patient. ${ }^{1}$ However, there is growing evidence that genetic knowledge may influence specific treatment recommendations. ${ }^{1}$ Hence, women newly diagnosed with breast cancer with high-risk clinical features are increasingly being offered genetic

The first two authors are joint first authors.

${ }^{1}$ Prince of Wales Clinical School, Faculty of Medicine, University of New South Wales, Sydney, Australia; ${ }^{2}$ Familial Cancer Service, Westmead Hospital, Westmead, Australia; ${ }^{3}$ Centre for Cancer Research, The Westmead Institute for Medical Research, University of Sydney, Westmead, Australia; ${ }^{4}$ Department of Medical Oncology, Hereditary Cancer Clinic, Prince of Wales Hospital, Randwick, Australia; ${ }^{5}$ Department of Obstetrics \& Gynaecology, Royal Women's Hospital, University of Melbourne, Parkville, Australia; ${ }^{6}$ School of Surgery, University of Western Australia, Crawley, Australia; ${ }^{7}$ School of Population Health, University of Western Australia, Crawley, Australia; ${ }^{8}$ Hunter Family Cancer Service, Waratah, Australia; ${ }^{9}$ Sydney Medical School-Northern, University of Sydney, Sydney, Australia; ${ }^{10}$ Royal North Shore Hospital, St Leonards, Australia; ${ }^{11}$ Monash Health, Melbourne, Australia;

${ }^{12}$ Familial Cancer Centre, Cabrini Health, Melbourne, Australia; ${ }^{13}$ Familial Cancer Centre, Peter MacCallum Cancer Centre, Melbourne, Australia; ${ }^{14}$ Genetic Health Queensland, Nambour General Hospital, Queensland, Australia; ${ }^{15}$ School of Psychology, University of Sydney, Sydney, Australia; ${ }^{16}$ Sir Peter MacCallum Department of Oncology, University of Melbourne, Melbourne, Australia. Correspondence: Bettina Meiser (b.meiser@unsw.edu.au) 
counseling and testing at diagnosis to allow appropriately timed treatment decisions (hereafter "treatment-focused genetic testing" (TFGT)). ${ }^{1}$ Apart from family history, these features include younger age ( $\leq 50$ years at diagnosis), triple-negative tumor, bilateral breast cancer, and/or Ashkenazi ethnicity. ${ }^{2}$

Currently the major contribution of identifying a mutation in a breast cancer predisposition gene is to facilitate the decision between breast conservation or mastectomy, with or without a contralateral mastectomy. The latter two options are associated with large reductions in the risk of ipsilateral and contralateral breast cancer. ${ }^{3-5}$ Two recent studies showed that mutation carriers who underwent bilateral mastectomy (BM) at the time of their first breast cancer diagnosis were less likely to die from breast cancer than women treated with unilateral mastectomy. ${ }^{3,4} \mathrm{BM}$ as a primary surgical treatment also circumvents the morbidity, cost, and recovery time of additional surgeries following delayed genetic testing and may bypass the morbidity, cost, and potentially negative impact of radiation treatment that is in most cases rendered unnecessary after BM. Breast conservation is also a reasonable approach for women, provided appropriate breast screening is available. Finally, carriers should consider risk-reducing bilateral salpingo-oophorectomy (RRBSO), which reduces the risk of ovarian cancer by up to 95\% ${ }^{6}$ and may halve the risk of breast cancer in premenopausal carriers, ${ }^{7}$ although this has recently been challenged. ${ }^{8}$

Several studies have shown that knowledge of BRCA1/2 mutation status increases uptake of BM..$^{9-12}$ More recently observed survival benefits associated with BM in carriers support the use of genetic testing early in the treatment trajectory. ${ }^{5,9}$ Furthermore, evidence for targeted therapies, such as poly(ADP-ribose) polymerase inhibitors ${ }^{13}$ and platinum agents, ${ }^{14}$ in BRCA1/2-associated breast cancer is accumulating, and the use of TFGT can direct such therapies. The increasing utility of TFGT will translate into additional pressures on genetics services and patient burden ${ }^{15}$ driving a need to develop alternative strategies to conventional models of genetic counseling that are both cost-effective and responsive to the time frames demanded in acute cancer management, without compromising informed consent.

This study compared the efficacy of providing brief written education (a pamphlet) about TFGT (intervention group (IG)) in preparing women for decision making about TFGT with conventional pretest, face-to-face genetic counseling (usualcare group (UCG)). Through a noninferiority trial, ${ }^{16}$ the primary hypothesis tested was that decisional conflict regarding testing choices would not be inferior in the IG compared with the UCG. Secondary aims were instead analyzed using superiority testing to assess the efficacy of brief education on knowledge of TFGT, breast cancer-specific worry, anxiety, depression, family involvement in decision making about TFGT, uptake of $\mathrm{BM}$ and RRBSO, and to compare the resource-based costs of the two methods of education about TFGT. Superiority testing was undertaken for secondary outcomes since any difference could be theoretically expected to occur in either direction; for example, removing the extra counseling session could lead to either negative experiences if women feel unprepared or, conversely, positive experiences if the burden or stress associated with another medical appointment is alleviated.

\section{Patients}

\section{MATERIALS AND METHODS}

Women were recruited through the breast clinics associated with eight participating FCCs in three Australian states. The methodology of this institutional review board-approved noninferiority randomized controlled trial is described in more detail elsewhere. ${ }^{17}$ In brief, women aged $18-49$ years and diagnosed with breast cancer (early invasive or ductal carcinoma in situ) were eligible if they were proficient in English and had either (i) a strong family history of breast and/or ovarian cancer $(\mathrm{FH}+)$ or (ii) no or minimal family history of breast and/or ovarian cancer (FH-) but a personal diagnosis of bilateral breast cancer and/or Ashkenazi Jewish ancestry and/or triple-negative breast cancer. Women were excluded if they had previously undergone genetic testing, a mastectomy, or breast radiotherapy, or if they were diagnosed with distant metastases or lobular in situ carcinoma. The relevant institutional review board at each participating site provided ethics approval.

\section{Procedure}

Eligible women were invited to participate by their treating breast surgeon. Participants were offered a choice of completing online or paper questionnaires. Following completion of the baseline questionnaire at time 1 (Supplementary Materials S1 online), participants were randomized to the IG or UCG. Women randomized to the IG were mailed a double-sided A4 trifold educational pamphlet (Supplementary Materials S2 online) developed on the basis of qualitative interviews with younger patients with breast cancer and pilot-tested before commencement of the trial with the target group..$^{18}$ Participants in both groups chose whether to have TFGT after receiving education about TFGT. TFGT results were available 8 working days from blood draw and was free of charge to the patient.

Approximately 1 week before their appointment to disclose results, women in the IG who elected to have TFGT participated in a telephone "intake" interview with a genetic counselor to ascertain a brief cancer family history. Participants allocated to the UCG also received an equivalent telephone "intake" interview with a genetic counselor before a full pretest, face-toface genetic counseling appointment.

Participants in both groups completed the questionnaire at time 2 (Supplementary Materials S3 online), within 1 week of receiving education about TFGT. All participants then received their test results in face-to-face appointments at their local FCC. The typical content of the results disclosure appointment has been described previously. ${ }^{17}$ Participants completed a questionnaire at time 3 (Supplementary Materials S4 online), 2 weeks following disclosure of TFGT results, and another questionnaire at time 4 (Supplementary Materials S5 online) 12 months after study enrollment (Figure 1). 


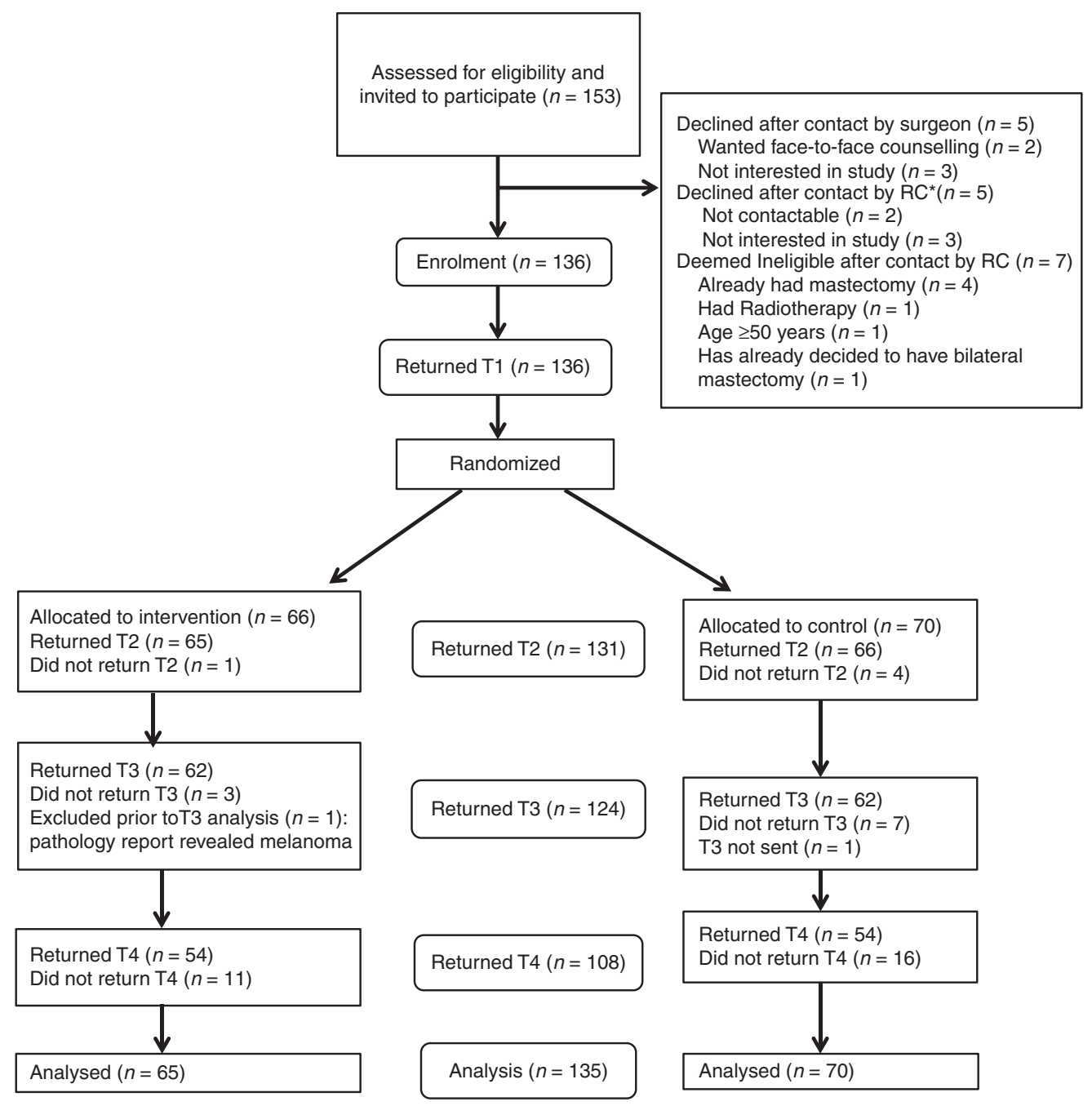

Figure 1 Flow of participants through the study.

\section{Measures}

Clinical information. Clinical information was collected directly from surgeons and/or pathology reports. Mutation carrier probability was calculated using the Breast and Ovarian Analysis of Disease Incidence and Carrier Estimation Algorithm. ${ }^{19}$

Health economic data. Surgeons and FCC staff recorded time spent (in minutes) during each patient consultation on pretest genetic counseling (UCG only) and results disclosure, including any necessary preparation time. Salary costs were collected for the different health professionals typically involved in each consultation type (averaged across different Australian states). Costs of developing the educational materials were also collected.

The following measures were included in patient questionnaires: time 1, sociodemographic data and measures (1) to (4); time 2, measures (1) to (4); time 3, measures (3) to (6); time 4, measures (3) to (5), (7), and (8).
1. Decisional conflict scale (DCS): Ten, three-point Likert scale items measured decisional conflict in relation to TFGT choice (scores range from 0 to 100 , with higher scores indicating more decisional conflict). ${ }^{20}$

2. Knowledge of TFGT: Ten true/false items were purposely designed and pilot-tested. Knowledge items are shown in Supplementary Figure S6 online. The total score was the number of correct responses.

3. Impact of Event Scale (IES): Fifteen items measured the frequency and severity of breast cancer-specific worry (range 0-75, with higher scores indicating more worry). ${ }^{21}$

4. Hospital Anxiety and Depression Scale (HADS): The 14-item HADS has two subscales measuring anxiety and depression. ${ }^{22}$ Scores range from 0 to 21 for each subscale, with higher scores indicating more anxiety or depression.

5. Test-Related Distress and Positive Experiences: Six items assessed distress (range 0-30) and four items positive experiences of genetic testing (range $0-30$ ), ${ }^{23}$ with higher scores indicating more distress and positive experiences. 
6. Family involvement in decision making about TFGT: One item asked women to indicate which family members were involved in the decision-making process about TFGT, if any.

7. Decision Regret Scale (DRS)_-Genetic Testing Choice: The five-item DRS was adapted to measure decision regret in relation to the decision to undergo TFGT. ${ }^{24}$ Higher scores indicate more regret (range 0-100).

8. DRS-Surgery Choice: Women completed two different versions of the DRS to measure decision regret relating to BM and RRBSO.

\section{Statistical power}

Decisional conflict was selected as the primary outcome and subject of noninferiority testing because of its demonstrated impact on actual decisions; for example, several studies that evaluated educational interventions show that lower levels of decisional conflict are associated with follow-through with decisions, and conversely, higher levels are associated with delays. ${ }^{17,20}$ Our protocol paper specified the noninferiority margin for the primary outcome variable, decisional conflict, as -10 units. ${ }^{17}$ That is, the IG was considered no worse than (noninferior to) the UCG if the 95\% confidence interval (CI) of the mean difference in DCS between the two groups lied wholly above -10 units. ${ }^{17}$ This trial aimed to obtain recruit 64 patients in each group (128 in total). This would provide $80 \%$ power to claim that the intervention is no worse than usual care (i.e., noninferior) with respect to Decisional Conflict Scale scores, assuming the true difference between groups was 0 and a standard deviation of 20 units. The power calculation was based on a $t$-test for a noninferiority margin of 10 units.

\section{Data analysis}

All analyses were conducted in IBM SPSS Statistics 21.0, and results were considered statistically significant when $P<0.05$. Variances were similar between the groups that were statistically compared. The primary outcome of decisional conflict and secondary outcome knowledge scores were assessed using linear regressions on scores after education, with scores before education added to the model as a covariate. This approach was prespecified in our published trial protocol and was selected over one using change scores because it has been argued that analyzing only change scores does not correct for any baseline differences due to the potential that those with low scores experienced more regression to the mean, and vice versa for those with initially high scores. ${ }^{25}$ Linear mixed effects models were used to assess secondary outcome variables, with multiple time points to allow for correlated and missing data. The goodness of fit for each model was compared using a first-order autoregressive covariance structure and an unstructured covariance structure. In all models the former was selected because it provided a better fit. Decision regret regarding TFGT, BM, and RRBSO were analyzed using linear regression, and two categorical outcomes (decision to undergo BM or RRBSO) were analyzed using binary logistic regression.

\section{Participant recruitment}

RESULTS

Recruitment took place from July 2010 to October 2012. Sociodemographic, medical history, and family history characteristics of the sample are detailed in Table 1. Of the 136 women enrolled, all were randomized and all opted to have TFGT. One woman was excluded from analyses subsequent to randomization because her pathology revealed benign proliferative disease; she was not found to have breast cancer.

Of the 135 analyzed, 77 (57.0\%) were categorized FH+, with the remainder having other risk factors for $B R C A 1 / 2$ mutations (FH-). Twenty (14.8\%) were identified as BRCA1/2 mutation carriers, 97 (71.9\%) had no mutation identified, and 18 (13.3\%) received a variant of unknown significance result. Of the 20 mutation carriers, $9(45.0 \%)$ were recruited as $\mathrm{FH}+$ and 7 (35.0\%), $3(15.0 \%)$, and $1(5.0 \%)$ on the basis of triple-negative histology, bilateral breast cancer, and Ashkenazi Jewish ancestry, respectively. The IG and UCG included 6 and 14 identified carriers, respectively.

\section{Decisional conflict regarding TFGT (primary outcome)}

The unadjusted mean, depicted in Supplementary Figure S6 online, for decisional conflict post education for the IG was 12.34 (95\% CI: 9.06-16.33) and for the UCG was $11.52(95 \%$ CI: 7.95-15.61), and when adjusting for baseline decisional conflict, this was 13.17 (95\% CI: 9.40-16.93) for the IG group and 10.719 (95\% CI: 7.01-14.42) for the UCG group. When controlling for baseline decisional conflict scores, study arm did not predict a statistically significant portion of variance in decisional conflict after education, indicating no difference between the IG and UCG ( $\beta=0.08$; 95\% CI: -2.87 to $7.76 ; P=0.36)$; a forest plot is shown in Figure 2. The lower limit of the 95\% CI, equivalent to a one-tailed $97.5 \%$ for the purposes of noninferiority, for the coefficient of the study arm variable (mean difference score) is greater than the noninferiority margin of -10 , suggesting noninferiority of the intervention. Table 2 depicts the mean differences and CIs for decisional conflict and all other psychological outcomes.

\section{Secondary psychological outcomes}

Supplementary Figures S8 to S14 online show the means for secondary outcomes. Study arm did not predict a significant proportion of variance in knowledge after education when controlling for baseline knowledge $(\beta=-0.06$; $95 \% \mathrm{CI}$ : $(-2.87$ to $7.76 ; P=0.49)$. There was no significant difference in breast cancer-specific worry scores between study arms $(d=0.19 ; P=0.27)$, and there was no significant study arm $\times$ time point interaction $(P=0.42)$.

There was no significant difference in HADS-Anxiety scores between study arms $(d=0.15 ; P=0.37)$, and there was no significant study arm $\times$ time point interaction $(P=0.21)$. There was no significant difference in HADS-Depression scores 
Table 1 Sociodemographic, medical, and family history characteristics of the usual-care and intervention groups $(N=135)$

\begin{tabular}{|c|c|c|c|c|}
\hline Variables & & $\begin{array}{c}\text { Intervention } \\
\text { group }(n=65) \\
\text { Mean (SD) }\end{array}$ & $\begin{array}{c}\text { Usual-care } \\
\text { group }(n=70) \\
\text { Mean (SD) }\end{array}$ & $\begin{array}{l}\text { Total sample } \\
\text { Mean (SD) }\end{array}$ \\
\hline Age at diagnosis, mean (SD) & Range: $25-50$ & $40.0(5.7)$ & $40.7(6.0)$ & $40.36(5.9)$ \\
\hline \multirow[t]{2}{*}{ Children } & Yes & $47(72.3)$ & $57(81.4)$ & $104(77.0)$ \\
\hline & No & $18(27.7)$ & $13(18.6)$ & $31(23.0)$ \\
\hline & Certificate & $8(12.3)$ & $15(21.4)$ & $23(17.0)$ \\
\hline & High school certificate & $17(26.2)$ & $22(31.4)$ & $39(28.9)$ \\
\hline & $\begin{array}{l}\text { Vocational college/college } \\
\text { degree/postgraduate degree }\end{array}$ & $32(49.2)$ & $29(41.4)$ & $61(45.2)$ \\
\hline Marital status & Married/living as married & $41(64.1)$ & $46(71.9)$ & $87(68.0)$ \\
\hline & Ashkenazi ancestry & $2(3.1)$ & $3(4.3)$ & $5(3.7)$ \\
\hline & Bilateral breast cancer & $4(6.2)$ & $9(12.9)$ & $13(9.6)$ \\
\hline & Triple-negative tumor & $20(30.8)$ & $20(28.6)$ & $40(29.6)$ \\
\hline \multirow[t]{3}{*}{ Carrier status } & Mutation carrier & $6(9.2)$ & $14(20.0)$ & $20(14.8)$ \\
\hline & No mutation found & $50(76.9)$ & $47(67.1)$ & $97(71.9)$ \\
\hline & $\begin{array}{l}\text { Variant of unknown } \\
\text { significance }\end{array}$ & $9(13.8)$ & $9(12.9)$ & $18(13.3)$ \\
\hline \multirow[t]{2}{*}{ Uni- versus bilateral cancer } & Unilateral cancer & $58(92.1)$ & $65(92.9)$ & $123(92.5)$ \\
\hline & Bilateral cancer & $5(7.9)$ & $5(7.1)$ & $10(7.5)$ \\
\hline \multirow[t]{2}{*}{ Nodal involvement ${ }^{a}$} & Yes & $17(32.1)$ & $28(42.4)$ & $45(37.8)$ \\
\hline & No & $36(67.9)$ & $38(57.6)$ & $74(62.2)$ \\
\hline
\end{tabular}

Data are $n(\%)$ unless otherwise indicated.

BOADICEA, Breast and Ovarian Analysis of Disease Incidence and Carrier Estimation Algorithm. ${ }^{22}$

aTumor size and grade refers to invasive carcinoma only.

between study arms $(d=0.03 ; P=0.86)$, and there was no significant study arm $\times$ time point interaction $(P=0.52)$.

There was no significant difference in test-related distress between study arms $(d=0.16 ; P=0.38)$, and there was no significant study arm $\times$ time point interaction $(P=0.50)$. There was no significant difference in test-related positive experiences between study arms $(d=0.11 ; P=0.55)$, and there was no significant study arm $\times$ time point interaction $(P=0.65)$.

There were no significant group differences in terms of family involvement in decision making about TFGT, with 40.3 and $32.3 \%$ in the IG and UCG reporting family involvement, respectively $\left(\chi^{2}=0.87 ; P=0.35\right)$. Participants named their spouse or partner, followed by their mother and then sister, most often as the family member who contributed to their decision-making process.

\section{Uptake of, and decision regret regarding, BM and RRBSO at 12 months}

At 12 months after enrollment, no differences were found between the IG and UCG in decision regret about having TFGT $(\beta=-0.04 ; P=0.69), \mathrm{BM}(\beta=0.02 ; P=0.87)$, or RRBSO $(\beta=-0.02 ; P=0.88)$. There were no significant differences between the two groups for uptake of BM: IG, $n=22(34.4 \%)$ and UCG, $n=25$ (36.4\%) (odds ratio = 1.16; 95\% CI: 0.57-2.38; $P=0.68$; Supplementary Table $\mathbf{S 1 5}$ online). The numerically higher uptake of RRBSO in the UCG $(n=11,17.2 \%)$ compared 
with the IG ( $n=6,9.4 \%)$ must be considered in the context of the uneven distribution of carriers in the UCG $(n=12)$ and IG $(n=6)$; however, there were no significant differences in uptake of RRBSO between the UCG and IG (odds ratio $=2.04$; 95\% CI: $0.71-5.90 ; P=0.19)$. A sensitivity analysis determined that controlling for mutation status did not affect either surgical logistic regression.

\section{Health economic comparison}

Table 3 compares the resource costs associated with each mode of education. The total cost per woman counseled in the IG was significantly lower (AUD\$90) compared with the UCG $($ AUD\$173) $(t(114)=5.93 ; P<0.001)$. Development costs and pilot-testing of the education pamphlet $(62 \mathrm{~h}$ of research staff time) amounted to AUD\$3,165 (AUD\$49/patient). If the developmental cost is included in the comparison, the cost per woman counseled in the IG is AUD\$137, which remains significantly lower than for the UCG $(t(114)=2.48 ; P=0.014)$.

Of the 135 participants included in the analyses, 109 also returned the 12-month questionnaire (follow-up rate of $80.7 \%$ ). No significant differences were found between participants who completed all questionnaires and those who did not in terms of baseline psychological characteristics, age, and educational level.

\section{DISCUSSION}

Given the climate of increasing cost constraints in health care in conjunction with the rapidly increasing demand for timely genetic testing in general, and TFGT in particular, ${ }^{26}$ the development of alternative cost-effective clinical service models is critical. Several alternatives to conventional face-to-face genetic counseling have been shown to be feasible, including videoconferencing, ${ }^{27}$ telephone genetic counseling, ${ }^{28}$ computer/ Internet-delivered education, ${ }^{29}$ and/or a combination of delivery methods. ${ }^{30}$ However, a dearth of data is currently available on the cost-effectiveness of these strategies. ${ }^{28}$
In this trial we tested an alternative streamlined model of service provision that incorporates brief written education about TFGT in place of conventional pretest genetic counseling. As hypothesized, we found that this was not inferior when compared with usual care in terms of the primary outcome of decisional conflict about testing choice. Also, as predicted, we found a significantly lower cost of providing brief education compared to face-to-face pretest genetic counseling. Thus this streamlined model of educating women about TFGT seems to be a cost-effective way of informing women newly diagnosed with breast cancer about TFGT while ensuring that women feel informed and supported in their decision making about genetic testing.

To our knowledge, this study is the first to include a substantial proportion of women offered TFGT based on features other than a family history of cancer. Women with no or minimal family history of cancer are unlikely to be aware that they are at risk of carrying a mutation and may experience higher levels of distress if identified as mutation carriers, compared with those with a strong family history. Women receiving a variant

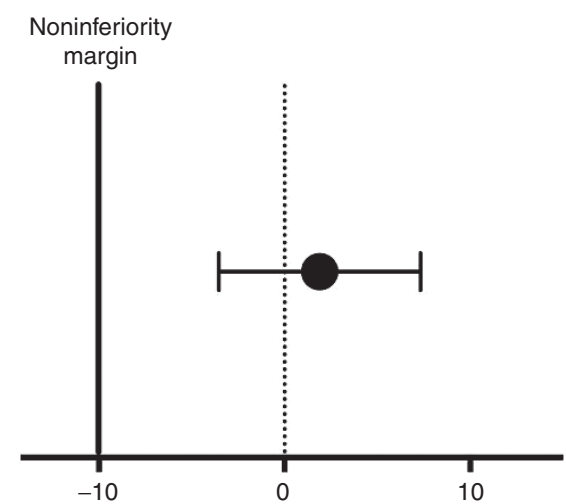

Figure 2 A forest plot depicting the prespecified noninferiority margin and the confidence interval around the difference between the intervention and usual-care groups.

Table 2 Mean difference (95\% confidence interval) for the primary and secondary outcomes at baseline, after education, and 2 weeks and 1 year after receipt of results

\begin{tabular}{|c|c|c|c|c|c|c|c|c|}
\hline \multirow[b]{2}{*}{ Outcome } & \multicolumn{2}{|r|}{ Baseline } & \multicolumn{2}{|r|}{ After education } & \multicolumn{2}{|c|}{ Two weeks after results } & \multicolumn{2}{|c|}{ One year after results } \\
\hline & $\begin{array}{l}\text { Patients } \\
(n)\end{array}$ & $\begin{array}{c}\text { Mean } \\
\text { difference (Cl) }\end{array}$ & $\begin{array}{l}\text { Patients } \\
(n)\end{array}$ & $\begin{array}{c}\text { Mean } \\
\text { difference (Cl) }\end{array}$ & $\begin{array}{l}\text { Patients } \\
(n)\end{array}$ & $\begin{array}{c}\text { Mean } \\
\text { difference (Cl) }\end{array}$ & $\begin{array}{l}\text { Patients } \\
(n)\end{array}$ & $\begin{array}{c}\text { Mean } \\
\text { difference (Cl) }\end{array}$ \\
\hline Decisional conflict & 135 & $-6.65(14.30-0.99)$ & 130 & $2.45(-2.87$ to 7.76$)$ & - & & - & \\
\hline IES & 135 & $1.94(-3.35$ to 7.23$)$ & 130 & $2.44(-2.89$ to 7.77$)$ & 124 & 4.49 (-0.90 to 9.88$)$ & 107 & $1.25(-4.38$ to 6.89$)$ \\
\hline HADS anxiety & 135 & $0.22(-1.30$ to 1.74$)$ & 130 & $1.09(-0.44$ to 2.63$)$ & 124 & $0.30(-1.25$ to 1.85$)$ & 107 & 0.77 (-0.84 to 2.39$)$ \\
\hline TRDP positive & - & & - & & 124 & 0.31 (-1.66 to 2.27$)$ & 107 & 0.77 (-1.30 to 2.84$)$ \\
\hline DRS TFGT & - & & - & & - & & 109 & $-0.75(-4.43$ to 2.92$)$ \\
\hline DRS BM & - & & - & & - & & 109 & $0.44(-4.92$ to 5.80$)$ \\
\hline DRS RRBSO & - & & - & & - & & 105 & $-0.52(-7.12$ to 6.08$)$ \\
\hline
\end{tabular}

Differences are calculated as intervention group minus usual-care group.

BM, bilateral mastectomy; CI, confidence interval; DRS, Decision Regret Scale; HADS, Hospital Anxiety and Depression Scale; IES, Impact of Event Scale; RRBSO, risk-reducing bilateral salpingo-oophorectomy; TFGT, treatment-focused genetic testing; TRDP, test-related distress and positive experiences. 
Table 3 Comparison of costs associated with each mode of information delivery

\begin{tabular}{|c|c|c|c|c|c|}
\hline \multirow[b]{2}{*}{ Resource } & \multirow[b]{2}{*}{$\begin{array}{l}\text { Performed } \\
\text { by }\end{array}$} & \multicolumn{2}{|c|}{ Intervention group $(n=65)$} & \multicolumn{2}{|c|}{ Usual-care group $(n=70)$} \\
\hline & & $\begin{array}{c}\text { Min/ } \\
\text { patient }\end{array}$ & $\begin{array}{c}\text { AUD\$/ } \\
\text { patient }{ }^{\text {a }}\end{array}$ & $\begin{array}{c}\text { Min/ } \\
\text { patient }\end{array}$ & $\begin{array}{l}\text { AUD\$/ } \\
\text { patient }^{\text {a }}\end{array}$ \\
\hline \multicolumn{6}{|l|}{ Pretest genetic counseling } \\
\hline Face-to-face consultation time & GC & - & - & $65(24.0)$ & $51(18.8)$ \\
\hline Total pretest genetic counseling time & & - & - & $114(64.2)$ & $89(50.4)$ \\
\hline Face-to-face consultation time & $G C+M G^{b}$ & $42(20.0)$ & $49(23.0)$ & $33(14.8)$ & $38(17.1)$ \\
\hline Total results disclosure time & & $92(49.6)$ & $89(40.6)$ & $90(70.0)$ & $83(57.3)$ \\
\hline Printing educational materials & & & 0.50 & & \\
\hline Total genetic counselling time & & $92(49.6)$ & $90(40.6)$ & $200(127.6)$ & $173(101.2)$ \\
\hline
\end{tabular}

Data are mean (SD) unless otherwise indicated.

GC, genetic counselor; MG, medical geneticist or oncologist with specialist training in familiar cancer.

aSalary costs for MGs, including on-costs, valid July 2013, averaged across Australian states (New South Wales and Victoria): \$2.461/min; salary costs for GCs (year 4/5, level 2, equivalent for experienced associate genetic counselor, including on-costs, valid July 2013, averaged across Australian states (New South Wales and Victoria): $\$ 0.785 /$ min. bAssumptions: $75 \%$ of consultation conducted by a GC, $25 \%$ by an MG.

of unknown significance result may also be more vulnerable to adverse psychological sequelae arising from TFGT, given that they continue to live with uncertainty. ${ }^{31}$

Before discussing the clinical implications of this study, its limitations should be mentioned. It was not possible to blind patients and clinicians at participating FCCs to randomization status, which may have introduced bias. All participants in this trial opted to receive their TFGT results, and it is therefore likely that participants are self-selected for interest in genetic testing and may not be fully representative of the larger group of younger women newly diagnosed with breast cancer. However, the study was not designed to measure uptake of TFGT among this larger group, and other studies have documented uptake rates, for example, among consecutively recruited samples. ${ }^{32}$ Nevertheless, study findings are generalizable to those women most likely to take up TFGT when offered. The majority of women (94.5\%) reported speaking mostly English at home, and thus generalizability to cultural and linguistic minorities is limited. Similarly, women were younger than 50 years of age, and might have greater genetic literacy; hence findings need to be replicated with older women. Moreover, multigene panel testing has been introduced in the nonacute setting in some countries, ${ }^{33}$ and the results reported may also need replicating in the multigene panel setting. In addition, the generalizability of findings to countries without national health services may be limited.

Finally, this study compared a particular model of genetic testing (no pretest genetic counseling followed by posttest face-to-face genetic counseling for all women) with traditional face-to-face pre- and posttest counseling. The clinical context is changing rapidly, and other models that involve a combination of face-to-face, telephone, and/or telehealth pre- and posttest counseling are being increasingly used alongside traditional face-to-face pre- and posttest genetic counseling ${ }^{34}$; even an invitation for women with ovarian cancer to have TFGT by mail has been implemented. ${ }^{8}$ However, the efficacy of these new models has not been rigorously evaluated, with the exception of pre- and posttesting telephone genetic counseling. ${ }^{28}$

In the US environment, much treatment-focused genetic testing already occurs outside of specialist genetics services, with access to and the extent of genetic counseling likely to vary between individual oncology units; outside of the United States, however, most genetic testing still occurs within the specialist genetics setting; this study provides support for a more targeted approach to this group of patients with a treatment decision influenced by a genetic test result. The number of carriers included in the study was small, and the study was not powered to detect differences by mutation status in important outcomes, such as regret after receipt of results. Finally, two trials were published after our protocol using a more conservative decisional criterion of four units, suggesting support for this more conservative margin. ${ }^{28,35}$ However, it is important to note that even using this criterion, our results would still be considered noninferior.

Our study also has important strengths, including a high follow-up rate at 12 months $(83.5 \%)$ and a lack of differences between those lost to follow-up and those retained; surgical outcome data were still available from file review for most of those lost to follow-up. Women were recruited from multiple FCCs across several states, each with different state-based health-care delivery; such heterogeneity of ascertainment indicates a high generalizability of the findings.

\section{Clinical implications}

The current model of pretest genetic counseling and testing provided by FCCs uses an approach derived from genetic testing for nonmalignant diseases, where knowledge of mutation status typically does not influence immediate treatment. By contrast, clinical contexts where genetic testing results can have direct implications for treatment and clinical management require 
streamlined service models if the results of germ-line genetic testing are to become fully integrated into cancer care. Recent surveys show that non-genetics health professionals, including surgeons, radiation oncologists, and medical oncologists, have positive attitudes and experiences with TFGT, ${ }^{36,37}$ indicating that involvement in the delivery of education should be acceptable to such health professionals. Findings from this study indicate that brief education, delivered in the context of a surgical/ oncological consultation, could be a safe and effective way of informing women newly diagnosed with breast cancer about TFGT. This service model could serve as a basis for developing similarly cost-effective models in other acute cancer settings, such as in the context of reflex tumor screening for mismatch repair deficiency associated with Lynch syndrome. ${ }^{38}$

\section{SUPPLEMENTARY MATERIAL}

Supplementary material is linked to the online version of the paper at http://www.nature.com/gim

\section{ACKNOWLEDGMENTS}

This trial was funded by a Priority-Driven Collaborative Research Grant that was jointly supported by Cancer Australia, Cancer Council Australia, and the National Breast Cancer Foundation (630405). B.M. was supported by a Career Development Fellowship Award Level 2 (1003921) and a Senior Research Fellowship Level B (1078523) from the National Health and Medical Research Council Australia. M.P. is currently supported by an Early Career Fellowship (ECF-15005) from the National Breast Cancer Foundation. The authors thank the patients who participated in this study, as well as the members of the TFGT Collaborative Group and the recruiting surgeons and staff involved at participating familial cancer clinics. The authors also gratefully acknowledge the assistance of the other health professionals and staff who were involved at each site. In addition, the authors thank Emma Healey for the calculation of BOADICEA scores, Mariana De Souza for research assistance, and Patrick Kelly, Gillian Heller, Val Gebski, and lan Marschner for statistical advice. Finally, the authors acknowledge the support and endorsement of this project by the Psycho-oncology Cooperative Research Group (PoCoG).

TFGT Collaborative Group: The additional members of the Treatment Focused Genetic Testing Collaborative Group are, in alphabetical order by group or institution: Cabrini Health-Melbourne (P. Gregory, L. Lipton, L. McKay, J. Senior); Calvary Centre for Palliative Care, Sydney (L. Lobb); Department of Medical Oncology, Prince of Wales Hospital (P. Crowe, A. Matthews, G. Neil, A. Parasyn, D. Thomson); Hereditary Cancer Clinic, Prince of Wales Hospital, Sydney (J. Duffy, L. Andrews, J. Gale); Monash Medical Centre, Melbourne (J. Fox, S. Hart, C. Smythe, M. White); Nambour Hospital, Nambour (L. Creighton, J. D'arcy, S. Grieve, E. Secomb); Peter MacCallum Cancer Centre, Melbourne (M. Henderson, J. O'Brien, C. Poliness); Royal Brisbane Hospital (A. Hattam, R. Susman, O. Ung,); Royal North Shore Hospital, Sydney (R. Dickson, K. Moore); St George Hospital, Sydney (P. Bastick, S. Inder, J. Lynch, P. Schwartz, R. Zia); The Poche Centre, Sydney (C. Mak, K. Snook, A. Spillane); University of Melbourne (J. Hopper);
Westmead Hospital, Sydney (M. Bowman, D. Cheung, S. Edirimanne, E. Edwards, E. Elder, J. French, D. Moon).

\section{DISCLOSURE}

The authors declare no conflict of interest.

\section{REFERENCES}

1. Trainer A, Lewis C, Tucker K, Meiser B, Friedlander M, Ward R. Treatmentfocused genetic assessment in breast cancer - the evolving role of the familial cancer services. Nat Rev Clin Oncol 2010;7:708-717.

2. Meiser B, Tucker K, Friedlander M, et al. Genetic counselling and testing for inherited gene mutations in newly diagnosed patients with breast cancer: a review of the existing literature and a proposed research agenda. Breast Cancer Res 2008; 10:216.

3. Metcalfe K, Gershman S, Ghadirian P, et al. Contralateral mastectomy and survival after breast cancer in carriers of BRCA1 and BRCA2 mutations: retrospective analysis. BMJ 2014;348:g226.

4. Evans DG, Ingham SL, Baildam A, et al. Contralateral mastectomy improves survival in women with BRCA1/2-associated breast cancer. Breast Cancer Res Treat 2013;140:135-142.

5. Kurian AW, Sigal BM, Plevritis SK. Survival analysis of cancer risk reduction strategies for BRCA1/2 mutation carriers. J Clin Oncol 2010;28:222-231.

6. Rebbeck TR, Kauff ND, Domchek SM. Meta-analysis of risk reduction estimates associated with risk-reducing salpingo-oophorectomy in BRCA1 or BRCA2 mutation carriers. J Natl Cancer Inst 2009;101:80-87.

7. Eisen A, Lubinski J, Klijn J, et al. Breast cancer risk following bilateral oophorectomy in BRCA1 and BRCA2 mutation carriers: an international casecontrol study. J Clin Onco/ 2005;23:7491-7496.

8. Plaskocinska I, Shipman H, Drummond J, et al. New paradigms for BRCA1/ BRCA2 testing in women with ovarian cancer: results of the Genetic Testing in Epithelial Ovarian Cancer (GTEOC) study. J Med Genet; e-pub ahead of print 12 May 2016.

9. Cortesi L, Razzaboni E, Toss A, et al. A rapid genetic counselling and testing in newly diagnosed breast cancer is associated with high rate of risk-reducing mastectomy in BRCA1/2-positive Italian women. Ann Onco/ 2014;25:57-63.

10. Graves KD, Peshkin BN, Halbert CH, DeMarco TA, Isaacs C, Schwartz MD. Predictors and outcomes of contralateral prophylactic mastectomy among breast cancer survivors. Breast Cancer Res Treat 2007;104:321-329.

11. Lokich E, Stuckey A, Raker C, Wilbur JS, Laprise J, Gass J. Preoperative genetic testing affects surgical decision making in breast cancer patients. Gynecol Oncol 2014;134:326-330.

12. Wevers $\mathrm{M}$, Aaronson $\mathrm{N}$, Verhoef $\mathrm{S}$, et al. Impact of rapid genetic counselling and testing on the decision to undergo immediate or delayed prophylactic mastectomy in newly diagnosed breast cancer patients: findings from a randomised controlled trial. Br J Cancer 2014;110:1081-1087.

13. Kaufman B, Shapira-Frommer R, Schmutzler RK, et al. Olaparib monotherapy in patients with advanced cancer and a germline BRCA1/2 mutation. J Clin Oncol 2015;33:244-250.

14. Byrski T, Huzarski T, Dent R, et al. Pathologic complete response to neoadjuvant cisplatin in BRCA1-positive breast cancer patients. Breast Cancer Res Treat 2014;147:401-405.

15. Katz SJ, Kurian AW, Morrow M. Treatment decision making and genetic testing for breast cancer: mainstreaming mutations. JAMA 2015;314:997-998.

16. European Medicines Agency Committee for Medicinal Products for Human Use. Guideline on the Choice of the Non-inferiority Margin. 2005. http://www. ema.europa.eu/docs/en_GB/document_library/Scientific_guideline/2009/09/ WC500003636.pdf. Accessed 1 September, 2016.

17. Watts KJ, Meiser B, Mitchell G, et al.; TFGT Collaborative Group. How should we discuss genetic testing with women newly diagnosed with breast cancer? Design and implementation of a randomized controlled trial of two models of delivering education about treatment-focused genetic testing to younger women newly diagnosed with breast cancer. BMC Cancer 2012;12:320.

18. Meiser B, Gleeson M, Watts K, et al. Getting to the point: what women newly diagnosed with breast cancer want to know about treatment-focused genetic testing. Oncol Nurs Forum 2012;39:E101-E111.

19. Antoniou AC, Cunningham AP, Peto J, et al. The BOADICEA model of genetic susceptibility to breast and ovarian cancers: updates and extensions. Br J Cancer 2008;98:1457-1466. 


\section{ORIGINAL RESEARCH ARTICLE}

20. O'Connor AM, Tugwell P, Wells GA, et al. Randomized trial of a portable, selfadministered decision aid for postmenopausal women considering long-term preventive hormone therapy. Med Decis Making 1998;18:295-303.

21. Thewes B, Meiser B, Hickie IB. Psychometric properties of the Impact of Event Scale amongst women at increased risk for hereditary breast cancer. Psychooncology 2001;10:459-468.

22. Ibbotson T, Maguire P, Selby P, Priestman T, Wallace L. Screening for anxiety and depression in cancer patients: the effects of disease and treatment. Eur J Cancer 1994;30A:37-40.

23. Cella D, Hughes $C$, Peterman A, et al. A brief assessment of concerns associated with genetic testing for cancer: the Multidimensional Impact of Cancer Risk Assessment (MICRA) questionnaire. Health Psychol 2002;21:564-572.

24. Brehaut JC, O'Connor AM, Wood TJ, et al. Validation of a decision regret scale. Med Decis Making 2003;23:281-292.

25. Vickers A, Altman D. Analysing controlled trials with baseline and follow up measurements. Br Med J 2001;323:1123-1124

26. James P, Mitchell G, Bogwitz M, Lindeman G. The Angelina Jolie effect: Media attention highlights the challenges faced by the rapidly developing field of familial cancer. Med J Austr 2013;199:646.

27. Zilliacus EM, Meiser B, Lobb EA, et al. Are videoconferenced consultations as effective as face-to-face consultations for hereditary breast and ovarian cancer genetic counseling? Genet Med 2011;13:933-941.

28. Schwartz MD, Valdimarsdottir HB, Peshkin BN, et al. Randomized noninferiority trial of telephone versus in-person genetic counseling for hereditary breast and ovarian cancer. J Clin Oncol 2014;32:618-626.

29. Green MJ, Biesecker BB, Mclnerney AM, Mauger D, Fost N. An interactive computer program can effectively educate patients about genetic testing for breast cancer susceptibility. Am J Med Genet 2001;103:16-23.
30. Sie AS, van Zelst-Stams WA, Spruijt L, et al. More breast cancer patients prefer BRCA-mutation testing without prior face-to-face genetic counseling. Fam Cancer 2014;13:143-151.

31. Wiggins S, Whyte P, Huggins $M$, et al. The psychological consequences of predictive testing for Huntington's disease. Canadian Collaborative Study of Predictive Testing. N Engl J Med 1992;327:1401-1405.

32. Schlich-Bakker KJ, Ausems MG, Schipper M, Ten Kroode HF, WárlámRodenhuis CC, van den Bout J. BRCA1/2 mutation testing in breast cancer patients: a prospective study of the long-term psychological impact of approach during adjuvant radiotherapy. Breast Cancer Res Treat 2008;109: 507-514.

33. Bradbury AR, Patrick-Miller L, Long J, et al. Development of a tiered and binned genetic counseling model for informed consent in the era of multiplex testing for cancer susceptibility. Genet Med 2015;17:485-492.

34. Trepanier AM, Allain DC. Models of service delivery for cancer genetic risk assessment and counseling. J Genet Couns 2014;23:239253.

35. Kinney AY, Butler KM, Schwartz MD, et al. Expanding access to BRCA1/2 genetic counseling with telephone delivery: a cluster randomized trial. J Natl Cancer Inst 2014;106:dju328.

36. Burcher S, Meiser B, Mitchell G, et al. Oncology health professionals' attitudes toward treatment-focused genetic testing for women newly diagnosed with breast cancer. Per Med 2013;10:431-440.

37. Douma KF, Meiser B, Kirk J, et al. Health professionals' evaluation of delivering treatment-focused genetic testing to women newly diagnosed with breast cancer. Fam Cancer 2015:14:265-272.

38. Vig HS, Wang $C$. The evolution of personalized cancer genetic counseling in the era of personalized medicine. Fam Cancer 2012;11:539-544. 